\title{
Article
}

\section{Effects of online intervention for depression on mood and positive symptoms in schizophrenia}

Moritz, Steffen, Schroeder, Johanna, Klein, Jan P, Lincoln, Tanja M, Andreou, Christina, Fischer, Anja and Arlt, Soenke

Available at http://clok.uclan.ac.uk/17032/

Moritz, Steffen, Schroeder, Johanna, Klein, Jan P, Lincoln, Tanja M, Andreou, Christina, Fischer, Anja ORCID: 0000-0002-7872-1292 and Arlt, Soenke (2016) Effects of online intervention for depression on mood and positive symptoms in schizophrenia. Schizophrenia Research, 175 (1-3). pp. 216-222. ISSN 09209964

It is advisable to refer to the publisher's version if you intend to cite from the work. http://dx.doi.org/10.1016/j.schres.2016.04.033

For more information about UCLan's research in this area go to http://www.uclan.ac.uk/researchgroups/ and search for <name of research Group>.

For information about Research generally at UCLan please go to http://www.uclan.ac.uk/research/

All outputs in CLoK are protected by Intellectual Property Rights law, including Copyright law. Copyright, IPR and Moral Rights for the works on this site are retained by the individual authors and/or other copyright owners. Terms and conditions for use of this material are defined in the policies page.

\section{CLoK}

Central Lancashire online Knowledge www.clok.uclan.ac.uk

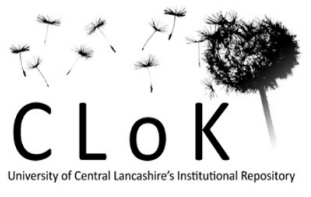




\title{
Effects of online intervention for depression on mood and positive
}

\section{symptoms in schizophrenia 25}

\author{
Steffen Moritz a,? ?,1, Johanna Schröder a,1, Jan Philipp Klein b, Tania M. Lincoln c, Christina Andreou a, \\ Anja Fischer d,2, Sönke Arlt a,2 \\ a University Medical Center Hamburg-Eppendorf, Department of Psychiatry and Psychotherapy, Martinistrasse 52, D-20246 Hamburg, Germany \\ b Department of Psychiatry and Psychotherapy, Lübeck University, Ratzeburger Allee 160, 23538 Lübeck, Germany \\ c Clinical Psychology and Psychotherapy, Institute of Psychology, University of Hamburg, 20146 Hamburg, Germany \\ d King's College London, Institute of Psychiatry, Psychology, and Neuroscience, Department of Health Psychology, Guy's Hospital, St Thomas Street, London SE1 9RT, \\ United Kingdom
}

F Previous presentation: The article has not been published before nor is under

consideration elsewhere.

[] Corresponding author.

E-mail address: moritz@uke.uni-hamburg.de (S. Moritz).

1 Split first authors.

2 Split senior authors.

Background: Depression is common in schizophrenia. Whereas the improvement of mood and self-esteem represents a subjective treatment priority for many patients, depression is rarely a primary target for clinical intervention. The present trial examined whether an online intervention for depression can ameliorate depressive symptoms in schizophrenia. Methods: A total of 58 individuals with schizophrenia were invited to participate in an online survey which encompassed the Center for Epidemiologic Studies-Depression Scale (CES-D, primary outcome), the Patient-Health-Questionnaire-9 (PHQ-9) and the Paranoia Checklist. Subsequently, telephone interviews were conducted to verify diagnostic status and assess symptoms (Positive and Negative Syndrome Scale, PANSS). Participantswere randomized either to the experimental condition (online depression intervention) or to a waitlist control condition. Three months after inclusion, a reassessment was carried out (self-report and telephone interviewblind for group condition). The trial was registered (registration: DRKS00007888).

Results: Participants in the treatment group showed a significant decline of depressive symptoms at a mediumto- large effect size, as assessedwith the CES-D and the PANSS depression item, in comparison to thewaitlist control group (completer (CC) and intention-to-treat analyses (ITT)). For the PHQ-9 (CC and ITT) and the PANSS distress subscale (CC only) significance was bordered at a medium effect size. Completion at the post-assessment after three months was $84 \%$. Discussion: Depression in schizophrenia is both underdiagnosed and undertreated. To reduce the large treatment gap in the disorder, low threshold strategies are urgently needed. Online treatment and bibliotherapy may represent valuable tools to address patients' needs beyond the treatment of the core positive syndrome.

\section{Introduction}

\subsection{Depression in schizophrenia}

Delusions and hallucinations (i.e., positive symptoms) are the defining features of psychosis and until recently represented the conventional target for the treatment of schizophrenia (Suzuki, 2011). In contrast, depressive symptoms in this disorder received comparatively little attention, presumably owing to historical and diagnostic reasons. Since Kraepelin (1899) who separated schizophrenia (then called dementia praecox) from affective disorders and more recently Schneider (1959) who confined the core of schizophrenia to positive features, depression is often regarded a negligible and secondary symptomatic feature of psychosis. Moreover, some phenomena that would be ounted as depressive symptoms in other disorders are labeled as negative symptoms in schizophrenia (e.g., social withdrawal) or even receive distinct diagnostic terms once a diagnosis of schizophrenia has been determined (Burckhardt, 2012; Kuck et al., 1992). For example, a mental state dominated by affective numbness is usually termed melancholia in depression but anhedonia in psychosis. Similarly, lack of drive (depression) is commonly relabeled as avolition in schizophrenia and again counted as a negative symptom. This, along with the longstanding preoccupation that psychosis is not amenable to understanding (Jaspers, 1973; Walker, 1991), may have contributed to the relative neglect of treatment of depressive symptoms in psychosis in the past and to the poor transfer of available psychological treatment options against depression in patients with psychosis.

\subsection{Reasons why depression is an important treatment target in schizophrenia}

A number of reasons existwhy depressive symptoms deserve greater attention in the treatment of psychosis. First, beginningwith Kasanin (1933), who coined the term schizoaffective disorder, clinicians increasingly acknowledge that affective symptoms coexist with psychosis. A review estimated that at least $50 \%$ of patients suffer from comorbid depression (Buckley et al., 2009), and many patients show single depressive symptoms such as low self-esteem (Freeman et al., 1998; Kesting and Lincoln, 2013; Moritz et al., 2010). Second, suicidality, a grave and life-threatening manifestation of depression, is frequent in psychosis and approximately $5 \%$ of patients commit suicide (Hor and Taylor, 2010). While some suicides are due to the influence of acoustic hallucinations, depression represents the best predictor for suicidality or selfharm behaviors in patients with schizophrenia (Fusar-Poli et al., 2014). Third, the implicit prevailing treatment paradigmposits that improving positive symptoms and insight will raise quality of life and reduce depression. Research suggests, however, that enhancement of insight can even paradoxically aggravate affective problems (Karow et al., 2008; Lincoln et al., 2007). Moreover, depression is not just a secondary consequence of having a severe mental disorder, it is also a frequent premorbid precursor of psychosis (Fusar-Poli et al., 2014). Fourth, depressive symptoms are often formulated by patients as their preferred target of treatment (Byrne et al., 2010; Hafner et al., 2013; Sterk et al., 2013; Moritz et al., in press-b).

\subsection{Poor efficacy of available treatment}

Notwithstanding the need to address depression in the therapy of psychosis as well as guidelines fostering psychotherapy in psychosis, many patients are still deprived of psychotherapy in general (Bechdolf and Klingberg, 2014; Shafran et al., 2009). If psychotherapy is sought at all, it often targets positive symptoms. Both pharmacological and psychological treatment approaches for psychosis are only partially effective in reducing depression. Antipsychotics yield a small effect size for the improvement of depressive symptoms in schizophrenia (Leucht et al., 2009). In fact, antipsychotic medication may even induce pharmacological depression (Naber and Karow, 2001). Augmentation with antidepressants has been shown ineffective for the treatment of depressive symptoms according to two metaanalyses (Kishi et al., 2013; Kishi and Iwata, 2014), underlining the need for non-pharmacological treatment options for depression in psychosis patients. Cognitivebehavioral therapy (CBT) yields a small to medium effect size (0.36) for the improvement of mood (Wykes et al., 2008). 
1.4. The present study

The present study explored whether a generic online intervention for depression administered in a non-clinical setting can reduce depressive

symptoms in patients with psychosis. We used a program called HelpID (developed by the novego AG) which is based on the CBT theoretical framework. Meta-analyses show that online interventions for depression exert a small-to-medium effect size in patients with depression when administered unguided (Cuijpers et al., 2011; Richards and Richardson, 2012, Johansson and Andersson, 2012).

Whether these effects also hold true for patients with comorbid depressive symptoms alongside other primary disorders, like obsessive-compulsive disorder, borderline personality disorder and schizophrenia, awaits to be established. While we hypothesize that HelpID will reduce depressive symptoms in psychosis, we were unable to make predictions with respect to the magnitude of the effect. Although self-help and online interventions have proven feasible in psychosis (Alvarez-Jimenez et al., 2014), a limiting factor could be that the online intervention under investigation is not adapted to the specific problems (e.g., stigma and self-stigma) and deficits (e.g., cognitive dysfunctions which may compromise translation of lessons/learning aims into everyday life) of psychotic patients. However, as patients with psychosis often do not receive specific treatment for depression, the potential of the program may be higher than in conventional (depression) populations who are usually not naïve about the contents of such programs. In linewith this, themagnitude of the effect of online interventions for depressionwasmoderate for a group of neurological patients in two recent studies of whom most had never received specific treatment for depression before (Fischer et al., 2015; Schröder et al., 2014). Based on the assumption that depressive symptoms and depression-related cognitions (e.g., worry thinking style, negative beliefs about the self, interpersonal sensitivity, sleep disturbance) play a causal role for the emergence of positive symptoms (Garety et al., 2001; Freeman and Garety, 2014; Lincoln et al., 2014),we also expected the treatment to impact on positive symptoms.

2. Method

2.1. Participants

Participants were primarily recruited via a database of former patients fromthe Department of Psychiatry and Psychotherapy at the University Medical Center Hamburg-Eppendorf (Germany, UKE). All former patients had previously given written informed consent to be re-contacted for future studies. While for most individuals a diagnosis of schizophrenia had been established on prior occasions, we additionally administered a diagnostic telephone interviewas a precondition for participation. We also contacted high-quality Internet forums (e.g. moderated, conveying evidence-based information) devoted to schizophrenia as an additional source to recruit study participants. The following inclusion criteria applied: age between 18 and 65 years, willingness to participate in two anonymous (Internetbased) surveys aswell as diagnostic telephone interviews that were scheduled threemonths apart, and a diagnosis of schizophrenia (as verified by telephone interview). Moreover, participants had to experience present subjective depressive symptoms and a willingness to undergo treatment for these symptoms (no formal cut-off was set). While presence of depressive symptoms was an explicit inclusion criterion, a diagnosis of major depression or dysthymic disorder was not. Severe suicidality led to exclusion. In these cases, participants were informed about help lines and treatment options. The trial was set up as an add-on intervention to care-as-usual: Participants were informed that the trial would not interfere with current treatments. For example, individuals were allowed to continue to take medication or see a physician. Interested individuals were directed to the baseline survey via a weblink. The survey was set up using questback ${ }^{\circledR}$, a software allowing to create online surveys. The study was anonymous (no name or postal address was requested; we did no store IP addresses were stored). Participants were informed that they would either immediately receive an online code allowing a free 3-month access to HelpID or would be allocated to a waitlist control condition. The latter group was promised full access to the program subsequent to the post-assessment (online survey and interview). Group allocation was carried out in random fashion subsequent to baseline assessment (i.e. following the interview) using an automated randomization plan with no stratification. The trial was registered at the Internet Portal of the German Clinical Trials Register (DRKS; DRKS00007888). The DRKSwas approved for the primary register in theWHO network and thusmeets the requirements of the International Committee of Medical Journal Editors (ICMJE).

\subsection{Procedure}

On the first page of the baseline survey, the rationale of the study as well as exclusion criteria were summarized. All participants provided electronic informed consent. Multiple log-ins via the same computer were prevented by means of "cookies". The survey consisted of the following parts: demographic section (e.g., gender, age), medical history (e.g., psychiatric diagnoses), assessment of psychopathology (see questionnaires section below), request for an email address (to match baseline and post-survey data), telephone number and contact information (e.g., preferred times for the interview) and whether prior responses had been correct. Following completion of the baseline assessment, interviewers tried to reach individuals at their preferred times to carry out the telephone interview (the assessments are described below). Telephone interviews were conducted blinded for group allocation and patients were explicitly reminded at the reassessment not to disclose which condition they had been allocated to. Following the blinded interview (see below) eligible participants were randomized (see above). Participantswere emailed by a researcher (JS) not involved in the interviews and informed aboutwhether they were allocated to the intervention group (a voucher providing access to the program was included along with further instructions) or the waitlist control group. Three months after the baseline interview, participants were contacted via email for participation in the postsurvey. Up to two reminders were dispatched if participants failed to complete the postassessment. For the post-survey, individuals were requested to first enter their email address to allow matching of baseline and post data. The postassessment consisted of the following parts: introduction, questionnaire on psychopathology (see below) and evaluation of the online intervention (if participants stated that they had logged in to the systemat least once). At the end, participants were asked whether they had made truthful responses and were given the opportunity to leave comments (e.g., if they experienced technical problems). They were then asked for their telephone number again and for their preferred times for the final interview. Following the final blinded interview, participants in the waitlist control group received an email containing a code giving full access to the program. All participants received a relaxation manual with audio files as an additional incentive.

\subsection{HelpID}

The Internet intervention Help ID (developed by novego AG, Seevetal, Germany) involves 12 weekly scheduled modules, which are derived from a pool of 17 modules (see below; 7 of these modules are mandatory). The individual set is composed according to the replies from a pre-assessment containing 60 questions which is performed prior to starting the program. Thus, every user receives a program tailored to their individual needs. Each module requires 45 to $60 \mathrm{~min}$ to complete and includes 14-19 pages of text. A video moderation 
leads through the program, which also includes interactive exercises and practice sheets, illustrations, photographs, animations and audios. For standard use of the program, motivational SMS (optional), email reminders and personal feedback on individual questions in a protected area are provided by the psychological team. For the present study however, no personal feedbackwas given aswewanted to evaluate the efficacy of a fully unguided treatment program. The program is based on a combination of therapeutic methods derived from $\mathrm{CBT}$, acceptance and commitment therapy (ACT) as well as systemic counseling and therapy also including relaxation audio files and music therapy, provided using an individualized algorithm considering gender-specific and symptom-related aspects, somatic variables (such as chronic back pain or cardiac arrhythmia) and potentially also postpartum depression. Additional modules on, for example, heart problems and post-partumdepressionwere presented if corresponding cue questions (here, on diagnosed heart deficits or the birth of a child in the last year) were affirmed. The algorithmalso took into account responses on the PHQ. Essential contents and goals of the program are to convey an understanding of depression by means of psychoeducation, the development of alternative viewpoints fostering activation in everyday life, strengthening social relationships as well as attention and relapse prevention exercises. The titles of the 17 modules are as follows (modules that are underlined are mandatory and administered to every individual): the way out of depression $\bullet$ getting started if you have the "blues" • depression • pleasant things in everyday life • learning to reward yourself • how to break thought spirals $\bullet$ together against depression $\bullet$ recognizing yourself $\bullet$ relaxation against depression $\bullet$ attention - made easy $\bullet$ learning to let go $\bullet$ doingmyself a favor $\bullet$ sun against murky thoughts $\bullet$ listen to your body $\bullet$ preventing relapse $\bullet$ therapeutic support $\bullet$ my heart and I. While seven of the modules were presented to each individual, five additional moduleswere selected according to individual responses to cue questions.

2.4. Questionnaires (online assessment)

Participants were required to complete three questionnaires. The survey proceeded only if all items had been responded to.

\subsubsection{Primary outcome}

The Center for Epidemiologic Studies-Depression Scale (CES-D) (Hautzinger and Brähler, 1993; Radloff, 1977) is a 20 itemquestionnaire covering depressive symptoms. In keeping with efforts to give patients' preferences and assessment greater consideration (Karamatskos et al., 2012), this scale represented the primary outcome of the study. The CES-D has both a good internal consistency and test-retest reliability $(r=0.81)$. Its validity has been confirmed against the Beck Depression Inventory (Beck and Steer, 1993). Items from the CES-D were mixed with those with the Paranoia Checklist.

\subsubsection{Secondary outcomes}

The Patient Health Questionnaire (PHQ-9; Kroenke et al., 2001) was assessed as an additional index of depression. The PHQ-9 is a selfreport instrument derived from the Primary Care Evaluation of Mental Disorders (PRIME-MD). Its nine items tap into the nine diagnostic criteria in the DSM-IV. Its psychometric properties can be judged as good with a sensitivity of 0.80 and a specificity of 0.92 (Gilbody et al., 2007). The Paranoia Checklist (Freeman et al., 2005) consists of 18 items tapping into subclinical as well as clinical signs of paranoid beliefs and suspiciousness. The psychometric properties are good (Freeman et al., 2005; Lincoln et al., 2010). The test-retest reliability of the online version is excellent (Moritz et al., in press-a; Moritz et al., 2014) and the scale shows good internal consistency and convergent validity (Lincoln et al., 2010). In our adaption of the scale, patients were required to rate the current symptom severity on a five-point Likert scale ranging from 1 (not at all) to 5 (extremely).

\subsection{Psychopathological interview}

A diagnosis of schizophrenia or schizoaffective disorderwas verified via telephone using the Mini-International Neuropsychiatric Interview (M.I.N.I.; Sheehan et al., 1998). Interviewers were blind to group status (intervention or control group). The M.I.N.I. has been successfully validated against other diagnostic tools (Sheehan et al., 1998). Symptomseverity was measured with the Positive and Negative Syndrome Scale (PANSS; Kay et al., 1989), which is considered the gold standard for the psychometric assessment of schizophrenia (Suzuki, 2011). The PANSS has good psychometric properties and is sensitive to change (Kay et al., 1989; Peralta and Cuesta, 1994; Santor et al., 2007). In order to avoid repetition of questions, both ratings were synthesized into one interview and not administered successively. Ratings followed semi-structured interview protocols. Before the trial, a rater training was held using several video demonstrations. In addition, the first interview was monitored by an experienced rater (JS). The same rater administered the interview for each individual patient to avoid rating biases. For the PANSS, we adopted a five-factor algorithm suggesting 


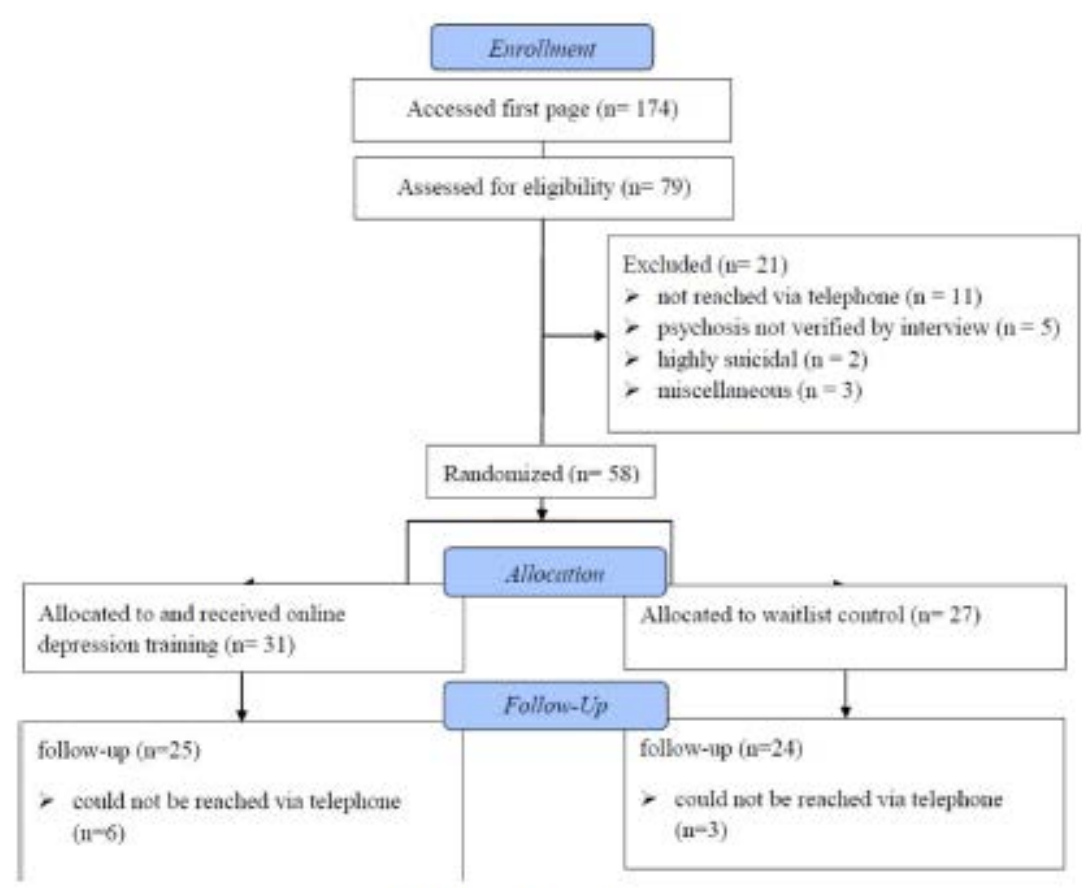

Fige 1. CONSORT chart diagram.

the following subscales: positive symptoms, negative symptoms, disorganization, excitement, and emotional distress (van der Gaag et al., 2006). The general item 6 (depression) served as an index for clinician-rated depression. However, as negative symptoms cannot be reliably assessed over the telephone, we did not calculate the PANSS negative subscale.

3. Results

A total of 174 participants accessed the first page of the survey; 105 participants provided their electronic informed consent. Seventy-nine participants completed the entire baseline survey. Of these, 21 were excluded for the reasons provided in the CONSORT chart (Fig. 1). Themain reason was that patients could not be reached via telephone $(n=11)$ because they either did not answer the phone $(n=6)$, did not leave a telephone number at all $(n=2)$, or left a wrong telephone number $(n=3)$. Thus, 58 participants fulfilling inclusion criteria were randomized to the two conditions.

\subsection{Baseline characteristics \& attrition}

The baseline characteristics of the sample are provided in Table 1. Except for a marginal difference on age, the groups did not differ on any baseline parameter. Depressive symptoms, as assessed with the PHQ-9, were moderate to severe. The PANSS total score signaled mild/ sub-acute symptomatology for most patients. The majority of participants were on medication (HelpID: $45.5 \%$ medication only, $9 \%$ psychotherapy only, $45.5 \%$ both; waitlist control: $61.1 \%$ medication only, $0 \%$ psychotherapy only, $38.9 \%$ both, $\left.\chi_{2}(1)=2.20, p=0.33\right)$. As shown in Table 1,most individuals fulfilled diagnostic criteria for current (comorbid) depression. The rest either were diagnosed with depressive episodes in the past, dysthymia or did not fulfill formal criteria for depression. After three months, $84 \%$ of the sample $(n=49$, HelpID: $n=25$, waitlist: $n=24$ ) were reached for the post-assessment (self-report and expert rating with the PANSS). Treatment status or concurrent treatment (e.g., lowered or enhanced dosage) did not change substantially between groups across time ( $\mathrm{p} \mathrm{N} 0.1)$.

\subsection{Statistical analyses}

In line with recommendations in the literature, we performed an ANCOVA with the pre-post difference score as the dependent variable and the respective baseline score as the covariate. This type of analysis accounts for regression to the mean and raises power of the analyses (Borm et al., 2007; Kenward and Roger, 1997).

\begin{tabular}{|c|c|c|c|c|c|}
\hline & \multicolumn{2}{|c|}{$\begin{array}{l}\text { HelpID depression } \\
\text { program }(n=31)\end{array}$} & \multicolumn{2}{|c|}{ Waitlist $(n=27)$} & Statistics $(t=56)$ \\
\hline \multicolumn{6}{|l|}{ Demogr aphic variables } \\
\hline Age in years & 38.19 & (11.78) & 4341 & $(8.42)$ & $t=1.91, p=0.06$ \\
\hline Cender (male/lemale) & $17 / 14$ & & $10 / 17$ & & $\chi^{2}(1)=184, p=0.17$ \\
\hline Years of formal school education & 1194 & (1.53) & 11.56 & $(145)$ & $t=0.97, p=0.34$ \\
\hline \multicolumn{6}{|l|}{ Psychopathalogy } \\
\hline Comar bid depression & $61 \%$ & & $64 x$ & & $\chi^{2}(1)=0.51, p=0.80$ \\
\hline PANSS total (corrected for negative symptoms) & 3339 & $(7,47)$ & 3593 & (9.14) & $\hat{t}=1.16, p=0.25$ \\
\hline CES-D & 5600 & (17.26) & 5626 & (16.53) & $t=0.06, p=0.95$ \\
\hline PHQ btal & 1055 & $(566)$ & 1244 & $(620)$ & $t=1.22, p=0.23$ \\
\hline Paranoia checklist & 3671 & (1807) & 3841 & (15.52) & $t=0.39, p=0.70$ \\
\hline
\end{tabular}




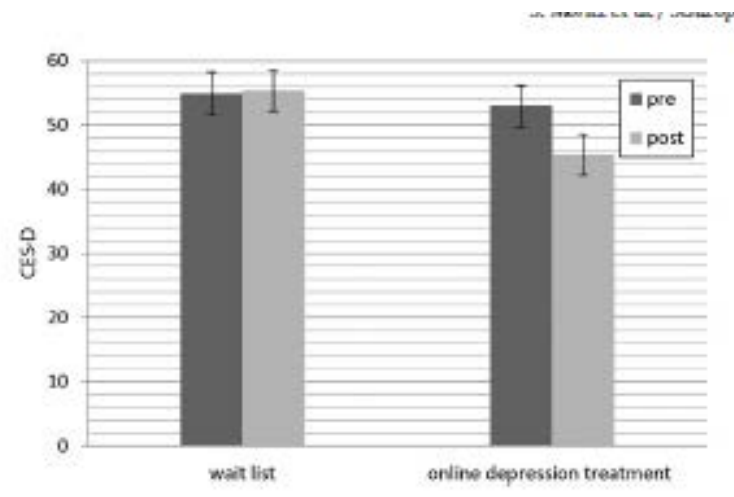

Fig. 2 CES-D: Significant decline of depressive symptams in the inter vention andition relative to the contral andition with a large effet size. Bars representstandard errors.

(CES-D) a significant difference emerged, $F(1,46)=9.84, p=0.003$, npartial2 $=0.176$ with a large effect size in favor of the intervention group relative to the waitlist group (see Fig. 2). Paired t-tests showed that symptoms decreased significantly in the treatment $(p=0.002)$ but not in the control condition $(p=0.822)$.

3.4. Secondary outcomes (complete cases)

For the PHQ-9 a trend in favor of the treatment group emerged at a medium effect size: ANCOVA: $F(1,46)=3.71, p=0.06, \eta$ partial $2=0.075$ (see Fig. 3). Paired t-tests showed that symptom decrease bordered on significance in the treatment $(p=0.052)$ but not in control group ( $p=0.788)$. As the PHQ-9 is a very short scale tapping into a heterogeneous set of symptoms, we conducted exploratory item-wise comparisonswhich revealed three differences in favor of the intervention group relative to the waitlist control group, particularly for self-

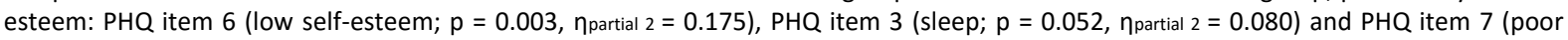
attention; $p=0.064$, $\eta_{\text {partial }} 2=0.073$ ). No group differences emerged on the Paranoia Checklist across time, $F(1,46)=0.13, p=0.91, \eta$ partial $2 b$ 0.001 (see Fig. 4). The PANSS depression item (general item 6) score decreased more strongly in the intervention group than in the waitlist control group at a medium-to-large effect size, $F(1,45)=5.07, p=0.029$, npartial $2=0.101$ (see Fig. 5). We then looked at the PANSS syndrome scales, whereby a marginally significant effect emerged for the PANSS distress subscale at amediumeffect size, $p=0.054, \eta$ partial $2=0.078$. For all other PANSS syndrome scores, the effects were non-significant (positive: $p=0.986$, disorganization: $p=0.172$, excitement: $p=0.245$ ). Exploratory item-wise analyses revealed a significant difference in favor of HelpID for blunted affect, which howeverwould have notwithstood correction for multiple testing (PANSS item N1), $F(1,46)=5.37, p=0.025, \eta_{\text {partial }} 2=0.104$.

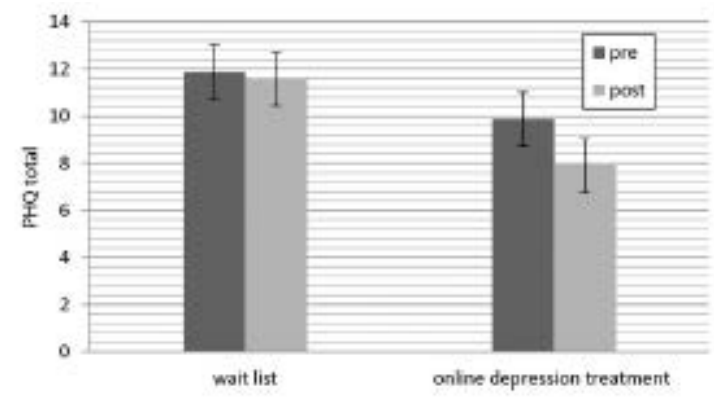

Fig. 3. PHQ-9: Decline (trend) of depressive symptarms in the intervention conditian relative to the control condition with a medium elfectsize. Bars represent stand ard errors

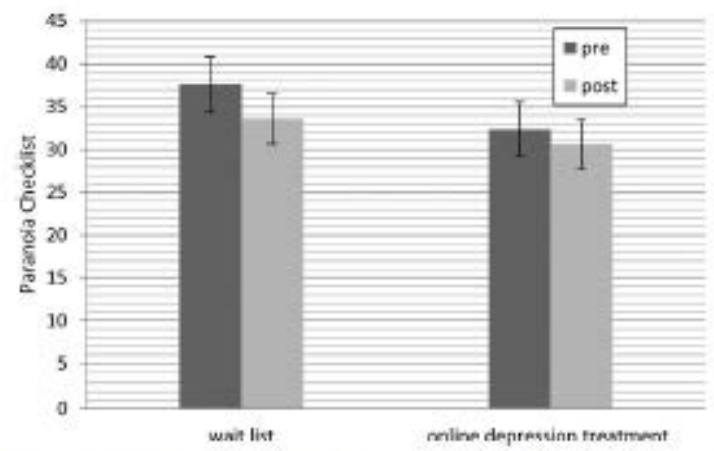

Fig.4. Pannoia checklist: Croups did not dirler across time an the dedine of paranoia and suspicousness Bars represent standard errars 
For intention-to-treat analyses considering all randomized patients, missing outcome data were imputed from information on the psychopathological and three demographic (age, gender, school education) indexes by the expectation-maximization (EM) algorithm, trimmed to fall between the minimumand maximumof possible values. This produced largely similar findings for all variables: $C E S-Q$ $\left(F(1,55)=12.023, p=0.001, \eta_{\text {partial }} 2=0.179\right)$, PANSS depression $\left(F(1,55)=4.356, p=0.042, \eta_{\text {partial } 2}=0.073\right), P H Q-9(F(1,55)=2.901, p=$ 0.094 , $\left.\eta_{\text {partial }} 2=0.05\right)$, PANSS positive $\left(F(1,55)=0.161, p=0.690\right.$, $\left.\eta_{\text {partial }}=0.003\right)$, PANSS disorganization $(F(1,55)=2.540, p=0.117, \eta$ partial $2=0.044)$, PANSS excitement $\left(F(1,55)=3.218, p=0.078\right.$, $\left.\eta_{\text {partial }} 2=0.055\right)$, PANSS distress $(F(1,55)=2.591, p=0.113, \eta$ partial

$2=0.045)$, Paranoia Checklist $\left(F(1,55)=0.061, p=0.806\right.$, $\left.\eta_{\text {partial } 2}=0.001\right)$.

\subsection{Subjective appraisal}

All individuals in the HelpID condition logged in to the program at least once. Three individuals acknowledged that they did not perform the exercises. Table 2 shows that more than almost two thirds treatment group would recommend the training to a friend, would use the program again, found the quality of the program good and regarded the program as being helpful in dealing with problems. Endorsement was lower for the following domains: individual help, useful for one's needs, satisfied with the degree of the help received by the program and satisfaction overall.

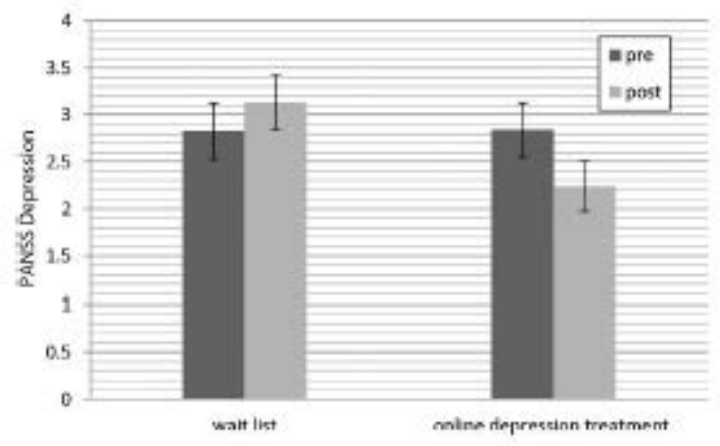

Fig 5. PANSS depressian item (g6): Significant dedine of depressive symptoms in the inter vention candition r dxive to the control candi bon at a medium-to-large effectsize. Bars represent standard errors

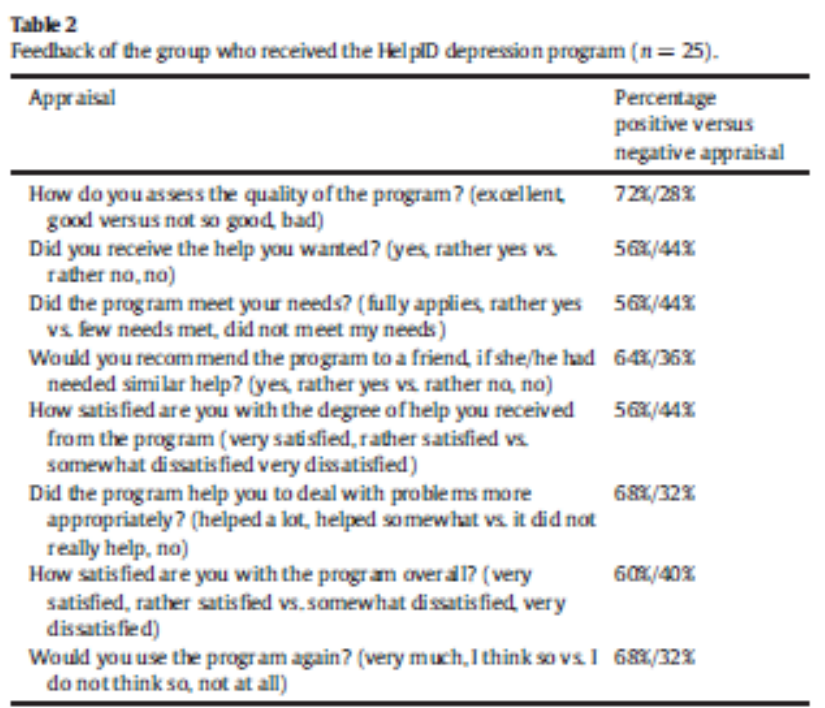

\subsection{Adherence}

A total of $16 \%$ of the sample used the program daily or almost daily, $28 \%$ used it once each week and $12 \%$ every two weeks. $24 \%$ used it once throughout the treatment period; $12 \%$ of the participants entered the programbut did not complete anymodule. On average, patients logged in 28.71 times ( $S D=46.67$; range: $2-232$ ).

\subsection{Test-retest reliability}

The three-month reliability was $r=0.62$ for the PANSS total score (corrected for the negative items), $r=0.79$ for the CES-Q, $r=0.70$ for the Paranoia Checklist and $r=0.62$ for the PHQ-9.

\section{Discussion}

Our study examined whether a generic and unguided online selfhelp intervention for depression, leads to an improvement of depressive symptoms in individualswith schizophrenia. Treatment of depression is of high importance in view of its prevalence in schizophrenia and because regulation of emotional problems represents a high treatment priority in patients (Byrne et al., 2010; Sterk et al., 2013; Moritz et al., in press-b). We combined the advantages of online research (e.g., economic implementation, facilitated access to patients of whom many would not have participated in an institutional treatment context) with that of a randomized-controlled clinical trial (RCT; diagnostic interview, external assessment with a gold-standard instrument, PANSS). We were able to recruit 58 individuals with a valid diagnosis 
of schizophrenia and reached $84 \%$ of the participants after threemonths. The majority of participants liked the program, found it helpful, would use it again and would recommend it to others, notwithstanding that the program did not address individual problems of patients with schizophrenia. This retrospective assessment was also mirrored by the main outcome: In line with our hypothesis, we observed a significant and large decline of depressive symptoms in the HelpID group relative to the waitlist control as assessed with the CES-D. While the results pointed into a similar direction (medium effect size) for the PHQ-9, the group differences only reached trend level for this scale. An exploratory post-hoc assessment of individual PHQ-9 items revealed group differences for self-esteem (PHQ item 6). For the PANSS depression item we found a significant difference with a medium-to-large effect size in favor of HelpID. The distress subscale, which also captures anxiety, revealed a trend in favor of HelpID. No significant differences were measured for the PANSS positive syndrome or Paranoia Checklist. Thus, it seems that reducing depression does not suffice to reduce positive symptoms as assessed by this scale. However, a longer follow-up investigation is currently planned to explorewhether a reduction of depressive symptomis followed by improvement on positive symptomsat a later time-point ("sleeper effect") as predicted by recent theoretical models (Freeman and Garety, 2014). Before drawing conclusions and suggesting ideas for future research, several limitations should be brought to the readers' attention. First, the sample sizewas rather small precluding elucidation of factors moderating outcome. Second, further follow-up studies are needed, ideally ones that include an active control condition to detectwhether improvement is sustained over time. Third, unlike conventional randomized controlled trials (RCTs), it is impossible to determine the screening-toinclusion ratio and to detect howthose participating in the trial differed from those who decided against it. Thus, despite encouraging evidence for the feasibility and efficacy of the approach its effectiveness needs to be replicated in a routine setting. Finally, while assessment with the PANSS is feasible for positive and depressive symptoms via the telephone, negative symptoms are hard to verify without face-to-face contact so that we could not compute scores for the PANSS negative subscale. To conclude, the study showed that an online intervention can improve depressive symptoms in individuals with psychosis. The magnitude of the effect was larger than was expected from prior studies in patients with primary depression (Spek et al., 2007). We speculate that this is due to the naivety of the recruited individuals concerning the contents of depression treatment which permitted its full potential to unfold. In contrast, many depressed patients undergoing online interventions for depression are usually familiar with standard face-to-face therapy thereby limiting surplus effects. Of note, the effect sizes were larger than those found for conventional treatment options for depression in psychosis such as antipsychotic (small effect) and antidepressant agents (negligible effect) and CBT (small-to-medium effect). We think that the larger "dosage" of the program (e.g., weekly sessions over 12 weeks) might have augmented the effect. Future studies should investigate whether guided interventions as well as incorporating elements addressing the needs and special problems of schizophrenia patients (e.g., stigma, socialwithdrawal, distrust, voice-hearing) can enhance the observed effects and generalize to psychotic symptoms.

Conflict of interest

None of the authors is affiliated with Novego ${ }^{\circledR}$ enterprise who created HelpID. The novego company was neither involved in the planning nor in analyzing the results of the trial. For the purpose of the trial, participants received free vouchers which were paid for by Novego.

Contributors

SteffenMoritz, Johanna Schröder, Jan Philipp Klein and TaniaM. Lincoln designed the study and wrote the protocol. Anja Fischer and Sönke Arlt were nvolved in the literature searches. Steffen Moritz, Johanna Schröder and Jan Philipp Klein undertook the statistical analysis. All authors substantially contributed to and have approved the final manuscript.

Role of funding source

The study did not receive external funding and was carried out using existing budget resources of the department.

Acknowledgement

We would like to thank Helena Mayer, Katharina Kolbeck, Nora Ramdani, Thies Lüdtke and Teresa Thöring for conducting the telephone interviews.

References

Alvarez-Jimenez, M., Alcazar-Corcoles, M.A., Gonzalez-Blanch, C., Bendall, S., McGorry,

P.D., Gleeson, J.F., 2014. Online, social media and mobile technologies for psychosis

treatment: a systematic review on novel user-led interventions. Schizophr. Res. 156, 96-106.

Bechdolf, A., Klingberg, S., 2014. Psychotherapy of schizophrenia: not a problem of evidence,

but a problem of implementation (Psychotherapie bei schizophrenen Störungen: Kein Evidenz-, sondern ein Implementierungsproblem). Psychiatr. Prax.

41, 8-10.

Beck, A.T., Steer, R.A., 1993. Beck Depression Inventory Manual. Psychological Corporation,

San Antonio.

Borm, G.F., Fransen, J., Lemmens,W.A., 2007. A simple sample size formula for analysis of

covariance in randomized clinical trials. J. Clin. Epidemiol. 60, 1234-1238.

Buckley, P.F., Miller, B.J., Lehrer, D.S., Castle, D.J., 2009. Psychiatric comorbidities and

schizophrenia. Schizophr. Bull. 35, 383-402.

Burckhardt, K., 2012. Why it is Difficult to Distinguish between Negative and Depressive

Symptomatology in Schizophrenia. University of Hamburg, Hamburg, Germany.

Byrne, R., Davies, L., Morrison, A.P., 2010. Priorities and preferences for the outcomes of

treatment of psychosis: a service user perspective. Psychosis 3, 210-217.

Cuijpers, P., Donker, T., Johansson, R.,Mohr, D.C., Van Straten, A., Andersson, G., 2011. Selfguided

psychological treatment for depressive symptoms: a meta-analysis. PLoS One

Fischer, A., Schröder, J., Vettorazzi, E., Wolf, O.T., Pöttgen, J., Lau, S., Heesen, C., Moritz, S.,

Gold, S.M., 2015. An online programme to reduce depression in patients with multiple

sclerosis: a randomised controlled trial. Lancet Psychiatry 2, 217-223.
Freeman, D., Garety, P., 2014. Advances in understanding and treating persecutory delusions:

a review. Soc. Psychiatry Psychiatr. Epidemiol. 49, 1179-1189.

Freeman, D., Garety, P., Fowler, D., Kuipers, E., Dunn, G., Bebbington, P., Hadley, C., 1998.

The London-East Anglia randomized controlled trial of cognitive-behaviour therapy

for psychosis. IV: Self-esteem and persecutory delusions. Br. J. Clin. Psychol. 37,

415-430.

Freeman, D., Garety, P.A., Bebbington, P.E., Smith, B., Rollinson, R., Fowler, D., Kuipers, E.,

Ray, K., Dunn, G., 2005. Psychological investigation of the structure of Ray, K., D

paranoia in

non-clinical population. Br. J. Psychiatry 186, 427-435.

Fusar-Poli, P., Nelson, B., Valmaggia, L., Yung, A.R., McGuire, P.K., 2014. Comorbid depressive

and anxiety disorders in 509 individuals with an at-risk mental state: impact on psychopathology and transition to psychosis. Schizophr. Bull. 40, 120-131. Garety, P.A., Kuipers, E., Fowler, D., Freeman, D., Bebbington, P.E., 2001. A cognitive model

of the positive symptoms of psychosis. Psychol. Med. 31, 189-195.

Gilbody, S., Richards, D., Brealey, S., Hewitt, C., 2007. Screening for depression in medical

settings with the patient health questionnaire (PHQ): a diagnostic metaanalysis.

J. Gen. Intern. Med. 22, 1596-1602.

Häfner, H., Maurer, K., an der Heiden,W., 2013. ABC schizophrenia study: an overview of

results since 1996. Soc. Psychiatry Psychiatr. Epidemiol. 48, 1021-1031.

Hautzinger, M., Brähler, M., 1993. Allgemeine Depressionsskala (ADS) [General Depression

Scale]. Hogrefe, Göttingen.

Hor, K., Taylor, M., 2010. Suicide and schizophrenia: a systematic review of rates and risk

factors. J. Psychopharmacol. 24, 81-90.

Jaspers, K., 1973. Allgemeine Psychopathologie [General Psychopathology]. Springer, Berlin 
(Germany)

Johansson, R., Andersson, G., 2012. Internet-based psychological treatments for depression.

Expert. Rev. Neurother. 12, 861-870.

Karamatskos, E.,Mulert, C., Lambert,M., Naber, D., 2012. Subjective wellbeing of patients

with schizophrenia as a target of drug treatment. Curr. Pharm. Biotechnol. 13, 1490-1499.

Karow, A., Pajonk, F.G., Reimer, J., Hirdes, F., Osterwald, C., Naber, D., Moritz, S., 2008. The

dilemma of insight into illness in schizophrenia: self- and expert-rated insight

quality of life. Eur. Arch. Psychiatry Clin. Neurosci. 258, 152-159.

Kasanin, J., 1933. The acute schizoaffective psychoses. Am. J. Psychiatr. 90, $97-126$.

Kay, S.R., Opler, L.A., Lindenmayer, J.P., 1989. The positive and negative syndrome scale

(PANSS): rationale and standardisation. Br. J. Psychiatry 155 (Suppl. 7), 5967.

Kenward, M.G., Roger, J.H., 1997. Small sample inference for fixed effects from restricted

maximum likelihood. Biometrics 53, 983-997.

Kesting, M.L., Lincoln, T.M., 2013. The relevance of self-esteem and selfschemas to persecutory

delusions: a systematic review. Compr. Psychiatry 54, 766-789.

Kishi, T., Iwata, N., 2014. Meta-analysis of noradrenergic and specific serotonergic antidepressant

use in schizophrenia. Int. J. Neuropsychopharmacol. 17, 343-354.

Kishi, T., Hirota, T., Iwata, N., 2013. Add-on fluvoxamine treatment for schizophrenia: an

updated meta-analysis of randomized controlled trials. Eur. Arch. Psychiatry Clin.

Neurosci. 263, 633-641

Kraepelin, E., 1899. Psychiatrie. Ein Lehrbuch für Studierende Und Aerzte [Psychiatry. A

Textbook for Students and Physicians]. J. A. Barth, Leipzig.

Kroenke, K., Spitzer, R.L., Williams, J.B., 2001. The PHQ-9: validity of a brief depression severity

measure. J. Gen. Intern. Med. 16, 606-613

Kuck, J., Zisook, S., Moranville, J.T., Heaton, R.K., Braff, D.L., 1992. Negative symptomatology

in schizophrenic outpatients. J. Nerv. Ment. Dis. 180, 510-515.

Leucht, S., Komossa, K., Rummel-Kluge, C., Corves, C., Hunger, H., Schmid, F., Asenjo Lobos,

C., Schwarz, S., Davis, J.M., 2009. A meta-analysis of head-to-head comparisons of

second-generation antipsychotics in the treatment of schizophrenia. Am.

J. Psychiatr. 166, 152-163.

Lincoln, T.M., Lüllmann, E., Rief, W., 2007. Correlates and long-term consequences of poor

insight in patients with schizophrenia. A systematic review. Schizophr. Bull. 33 ,

1324-1342.

Lincoln, T.M., Peter, N., Schafer, M., Moritz, S., 2010. From stress to paranoia: an experimental

investigation of the moderating and mediating role of reasoning biases.

Psychol. Med. 40, 169-171.

Lincoln, T.M.,Mobius, C., Huber,M.T., Nagel,M.,Moritz, S., 2014. Frequency and correlates

of maladaptive responses to paranoid thoughts in patients with psychosis compared

to a population sample. Cogn. Neurodyn. 19, 509-526.

Moritz, S., Veckenstedt, R., Randjbar, S., Vitzthum, F., Karow, A., Lincoln,

T.M., 2010. Course

and determinants of self-esteem in people diagnosed with schizophrenia during psychiatric

treatment. Psychosis 2, 144-153.

Moritz, S., Voigt, M., Kother, U., Leighton, L., Kjahili, B., Babur, Z., Jungclaussen, D.,

Veckenstedt, R., Grzella, K., 2014. Can virtual reality reduce reality distortion? Impact of performance feedback on symptom change in schizophrenia patients. J. Behav.

Ther. Exp. Psychiatry 45, 267-271.

Moritz, S., Endlich, L.,Mayer, H., Andreou, C., Ramdani, N., Petermann, F. \& Balzan, R. P. (in

press-a). The benefits of doubt: cognitive bias correction reduces hasty decisionmaking

in schizophrenia. Cogn. Ther. Res.

Moritz, S., Berna, F., Jaeger, S., Westermann, S. \& Nagel, M. (in press-b). The customer is

always right? Subjective target symptoms and treatment preferences in patients with psychosis. Eur. Arch. Psychiatry Clin. Neurosci.

Naber, D., Karow, A., 2001. Good tolerability equals good results: the patient's perspective.

Eur. Neuropsychopharmacol. 11 (Suppl. 4), S391-S396

Peralta, V., Cuesta, M.J., 1994. Psychometric properties of the positive and negative syndrome

scale (PANSS) in schizophrenia. Psychiatry Res. 53, 31-40.

Radloff, L.S., 1977. The CES-D scale: a self-report depression scale for research in the general

population. Appl. Psychol. Meas. 1, 385-401.

Richards, D., Richardson, T., 2012. Computer-based psychological treatments for depression:

a systematic review and meta-analysis. Clin. Psychol. Rev. 32, 329-342.

Santor, D.A., Ascher-Svanum, H., Lindenmayer, J.P., Obenchain, R.L., 2007. Item response

analysis of the positive and negative syndrome scale. BMC Psychiatry 7, 66.

Schneider, K., 1959. Clinical Psychopathology. Grune and Stratton, New York.

Schröder, J., Brückner, K., Fischer, A., Lindenau, M., Köther, U., Vettorazzi,

E., Moritz, S.,

2014. Efficacy of a psychological online intervention for depression in people with

epilepsy: a randomized controlled trial. Epilepsia 55, 2069-2076.

Shafran, R., Clark, D.M., Fairburn, C.G., Arntz, A., Barlow, D.H., Ehlers, A., Freeston, M.,

Garety, P.A., Hollon, S.D., Ost, L.G., Salkovskis, P.M., Williams, J.M.G., Wilson, G.T.,

2009. Mind the gap: improving the dissemination of CBT. Behav. Res. Ther.

902-909.

Sheehan, D.V., Lecrubier, Y., Sheehan, K.H., Amorim, P., Janavs, J., Weiller, E., Hergueta, T.

Baker, R., Dunbar, G.C., 1998. The Mini-International Neuropsychiatric Interview

(M.I.N.I.): the development and validation of a structured diagnostic psychiatric interview

for DSM-IV and ICD-10. J. Clin. Psychiatry 59 (Suppl. 20), 22-33.

Spek, V., Cuijpers, P., Nyklicek, I., Riper, H., Keyzer, J., Pop, V., 2007. Internet-based cognitive

behaviour therapy for symptoms of depression and anxiety: a meta-analysis.

Psychol. Med. 37, 319-328.

Sterk, B., Winter van Rossum, I., Muis, M., de Haan, L., 2013. Priorities, satisfaction and

treatment goals in psychosis patients: an online consumer's survey.

Pharmacopsychiatry 46, 88-93.

Suzuki, T., 2011. Which rating scales are regarded as 'the standard' in clinical trials for

schizophrenia? A critical review. Psychopharmacol. Bull. 44, 18-31.

Van der Gaag, M., Hoffman, T., Remijsen, M., Hijman, R., de Haan, L., van Meijel, B., van

Harten, P.N., Valmaggia, L., de Hert, M., Cuijpers, A., Wiersma, D., 2006. The fivefactor

model of the positive and negative syndrome scale II: a ten-fold crossvalidation of a revised model. Schizophr. Res. 85, 280-287.

Walker, C., 1991. Delusion: what did jaspers really say? Br. J. Psychiatry 159 (Suppl. 14),

94-103.

Wykes, T., Steel, C., Everitt, B., Tarrier, N., 2008. Cognitive behavior therapy for schizophrenia:

effect sizes, clinical models, and methodological rigor. Schizophr. Bull. 34, 523-537.

S. Moritz et al. / Schizophrenia Research xxx (2016) xxx-xxx 7 